\title{
Conformable Diferensiyel Denklemlerin Genelleştirilmiş Kudryashov Yöntemiyle Tam Çözümleri
}

\author{
Arzu AKBULUT ${ }^{* 1(D,}$, Melike KAPLAN 2 (1) \\ ${ }^{1}$ Eskişehir Osmangazi Üniversitesi, Fen-Edebiyat Fakültesi, Matematik-Bilgisayar Bölümü, 26040, Eskişehir, \\ Türkiye \\ ${ }^{2}$ Kastamonu Üniversitesi, Fen-Edebiyat Fakültesi, Matematik Bölümü, 37150, Kastamonu, Türkiye
}

(Alınış / Received: 01.01.2021, Kabul / Accepted: 05.02.2021, Online Yayınlanma / Published Online: 15.04.2021)

\author{
Anahtar Kelimeler \\ Tam çözümler, \\ Genelleştirilmiş Kudryashov \\ metot, \\ Sembolik hesaplama, \\ Conformable zaman kesir \\ mertebeli üçüncü \\ mertebeden modifiye $\mathrm{KdV}$ \\ denklemi, \\ Conformable zaman kesir \\ mertebeli Boussinesq \\ denklemi
}

\begin{abstract}
Özet: Lineer olmayan conformable diferensiyel denklemler matematiksel fizikte önemli bir yere sahiptir. Bu denklemlerin tam çözümlerinin elde edilmesi, son yıllarda oldukça ilgi çeken bir çalışma alanı olarak karşımıza çıkmaktadır. Bu makalede, conformable üçüncü mertebeden modifiye KdV denklemi ve conformable Boussinesq denkleminin tam çözümleri genelleştirilmiş Kudryashov yöntemi kullanılarak bulunmuştur. Bu yöntem, lineer olmayan conformable denklemlerin tam çözümlerini elde etmede kullanılan etkili bir yöntemdir. Bu çalışmadaki bütün hesaplamalar Maple paket programı kullanılarak yapılmıș ve elde edilen çözümler denklemlerde yerine konularak doğruluğu teyit edilmiştir. Ayrıca elde edilen çözümlerin grafiklerine de yer verilmiştir. Elde edilen çözümler, matematiksel fizik ve mühendislik alanlarında önemli kullanım alanlarına sahip olma potansiyeline sahiptirler.
\end{abstract}

\section{Exact Solutions of Conformable Differential Equations Using Generalized Kudryashov Method}

\section{Keywords}

Exact solutions,

Generalized Kudryashov method,

Symbolic computation, Conformable time fractional third order modified KdV equation,

Conformable time fractional Boussinesq equation

\begin{abstract}
Nonlinear conformable differential equations have an important place in mathematical physics. Recently, the search for exact solutions of these equtions has been an appealing field for most scientists. In this work, exact solutions of the conformable third-order modified $\mathrm{KdV}$ equation and conformable Boussinesq equation founded by using the generalized Kudryashov method. This method is an effective method to acquire exact solutions of nonlinear conformable equations. All calculations in this study have been made and checked back with the aid of the Maple packet program. Also, the graphical representation of the obtained solutions is given. The obtained solutions in this manuscript have the potential to be useful in mathematical physics and engineering.
\end{abstract}

\section{Giriș}

Son yllarda, plazma fiziği, matematiksel biyoloji, akışkanlar mekaniği, fiber optik, türbülans teorisi, soliton teori ve matematiksel fizik alanlarında lineer olmayan oluşum denklemleri üzerine yapılan çalışmalar büyük önem kazanmıştır. Lineer olmayan kısmi diferensiyel denklemlerin analitik çözümleri, modellemiş oldukları olayın yorumlanmasına katkı sağlamaktadır. $\mathrm{Bu}$ sebeple araștırmacılar bu denklemlerin çözümlerinin elde edilmesi için Maple, Matlab ve Mathematica gibi paket programlar yardımıyla çeşitli yöntemler geliştirmişlerdir. Bunlardan bazıları ters saçılım metodu [1], homojen denge metodu [2], Hirota bilineer metot [3, 4], üstel fonksiyon metodu [5], trial denklem metodu [6], genişletilmiş trial denklem metodu [7], sine-Gordon açlım metodu $[8,9]$ ve genelleştirilmiş Kudryashov metodu [10] olarak sıralanabilir. Bu yöntemler içinden en etkilisi şeklinde bir tanımlama yapmak mümkün değildir. Bu sebeple de bu alan popülerliğini korumaya devam etmektedir.

$\mathrm{Bu}$ çalışmanın amacı genelleştirilmiş Kudryashov yöntemi kullanılarak conformable üçüncü mertebeden modifiye KdV denklemi ve conformable Boussinesq denkleminin farklı analitik çözümlerini elde etmektir. Bir başka deyişle, bu çalışmada elde 
edilen çözümleri diğer çalışmalarda elde edilenlerle kıyaslarsak, parametrelerin uygun seçimi ile diğer çalışmalarda benzer çözümler elde edilebilecek iken, bu çalışmada elde edilen çözümlerin yine farklı olduğu görülür. Ayrıca genelleştirilmiş Kudryshov yöntemi diğer yöntemlere kıyasla uygulanması daha pratik bir yöntemdir.

Makalenin geri kalan bölümleri şu şekilde organize edilmiştir. İkinci bölümde genelleştirilmiş Kudrayshov yöntemi adım adım tanıtılmıştır. Üçüncü bölümünde bu yöntemin uygulamalarına yer verilmiştir. Dördüncü bölümde ise tartışma ve sonuçlara yer verilmiştir.

\section{Genelleştirilmiş Kudryashov Metot}

$\mathrm{Bu}$ bölümde, lineer olmayan kısmi diferensiyel denklemlerin tam çözümlerinin bulunması için genelleştirilmiş Kudryashov metodunu vereceğiz. Bunun için öncelikle conformable türev ile ilgili gerekli bilgiler vereceğiz.

\section{Conformable kesirli türev ve özellikleri}

$f:[0, \infty) \rightarrow R \quad$ şeklinde fonksiyon olsun. $\alpha$. mertebeden $f$ fonksiyonunun conformable kesirli türevi $t>0, \alpha \in(0,1]$ olmak üzere;

$$
\left(T_{\alpha} f\right)(t)=\lim _{\varepsilon \rightarrow 0} \frac{f\left(t+\varepsilon t^{1-\alpha}\right)-f(t)}{\varepsilon}
$$

şeklinde tanımlanır ve aşağıdaki özellikleri sağlar.

$T_{\alpha}(a f+b g)=a\left(T_{\alpha} f\right)+b\left(T_{\alpha} g\right), a, b \in R$

$T_{\alpha}\left(t^{p}\right)=p t^{p-\alpha}, p \epsilon R$

$T_{\alpha}(f(t))=0, f(t)=\lambda$ sabit fonksiyondur.

$T_{\alpha}(f g)=f T_{\alpha}(g)+g T_{\alpha}(f)$

$T_{\alpha}(f / g)=\frac{g\left(T_{\alpha} f\right)-f\left(T_{\alpha} g\right)}{g^{2}}$

Ek olarak $f$ diferensiyellenebilir bir fonksiyon ise $T_{\alpha}(f)(t)=t^{1-\alpha} \frac{d f}{d t}(t)$ olur [11].

Teorem: $\quad f, g:[0, \infty) \rightarrow R, \quad \alpha . \quad$ mertebeden diferensiyellenebilir fonksiyonlar olsunlar. 0 halde aşağıdaki zincir kuralı sağlanır [11].

$$
T_{\alpha}(f \circ g)(t)=t^{1-\alpha} g^{\prime}(t) f^{\prime}(g(t)) \text {. }
$$

Şimdi genelleştirilmiş Kudryashov metodu verelim [12]. Genel olarak lineer olmayan kısmi diferensiyel denklemler

$$
\mathrm{P}\left(\mathrm{u}, \frac{\partial^{\alpha} u}{\partial t^{\alpha}}, \frac{\partial u}{\partial x}, \frac{\partial^{2 \alpha} u}{\partial t^{2 \alpha}}, \frac{\partial^{2} u}{\partial x^{2}}, \ldots\right)=0
$$

şeklinde olsun. Burada $\mathrm{P}$, yüksek mertebeden kısmi türevler ve lineer olmayan terimler içeren $u(x, y, z, t)$ nin bir polinomudur.

Genelleştirilmiş Kudryashov metodunun ana adımlarını aşağıda vereceğiz:

Adım 1: Denklemlerin dalga çözümlerini bulabilmek için öncelikle aşağıdaki dalga dönüşümü kullanılır.

$$
\mathrm{u}(\mathrm{x}, \mathrm{t})=\mathrm{u}(\xi), \xi=\mathrm{x}-l \frac{t^{\alpha_{1}}}{\alpha_{1}}
$$

Denklem (2) kullanıldığında, Denklem (1) aşağıdaki formda verilen lineer olmayan adi diferensiyel denkleme dönüşür.

$$
H\left(u, u^{\prime}, u^{\prime \prime}, \ldots,\right)=0
$$

Buradaki türev, $\xi$ ye göre türevi gösterir.

Adım 2: Genelleştirilmiş Kudrayshov metoduna göre, denklem (3) ün çözümü aşağıdaki rasyonel formda aranacaktır.

$$
u(\xi)=\frac{\sum_{i=0}^{N} \mathrm{a}_{\mathrm{i}} Q^{\mathrm{i}}(\xi)}{\sum_{j=0}^{M} \mathrm{~b}_{\mathrm{j}} Q^{\mathrm{j}}(\xi)}
$$

Burada $a_{i}(i=0,1, \ldots, n), \quad b_{j}(j=0,1, \ldots, m)$ sabitlerdir ve $\mathrm{a}_{\mathrm{N}} \neq 0, \mathrm{~b}_{\mathrm{M}} \neq 0$. Ayrica $Q=\mathrm{Q}(\xi)$

$$
\frac{d Q}{d \xi}=Q^{2}(\xi)-Q(\xi)
$$

şeklindeki adi diferensiyel denklemi sağlar. Denklem (5) in çözümünün;

$$
Q(\xi)=\frac{1}{1+A e^{\xi}}
$$

şeklinde olduğu açıtır ve $A$ integral sabitidir.

Adım 3: Denklem (4) teki pozitif tamsayı olan N ve M homojen denge metodu kullanilarak bulunur. Bir başka deyișle, en yüksek mertebeden türevli terim ile en yüksek dereceden lineer olmayan terimlerin derecelerinin dengelenmesiyle elde edilir.

Adım 4: Denklem (5) yardımıyla Denklem (4), Denklem (3) te yerine yazılırsa, $Q$ nun $R(Q)$ polinomu elde edilir. $\mathrm{R}(\mathrm{Q})$ nun bütün katsayıları sıfıra eşitlenirse, cebirsel denklemler sistemi elde edilir. Maple yardımıyla bu sistem çözülürse, $\mathrm{a}_{\mathrm{i}}(\mathrm{i}=$ $0,1, \ldots, n), \quad b_{j}(j=0,1, \ldots, m)$ değerleri bulunur. Son olarak, Denklem (5) ve bulunan değerler Denklem (4) te yerlerine yazılırsa ele alınan denklemin tam çözümleri bulunur [13]. 


\section{Uygulamalar}

\subsection{Conformable Üçüncü Mertebeden Modifiye KdV Denklemi}

Conformable üçüncü mertebeden modifiye $\mathrm{KdV}$ denklemi

$$
\mathrm{D}_{\mathrm{t}}^{\alpha} u+p u^{2} \mathrm{u}_{\mathrm{x}}+q \mathrm{u}_{\mathrm{xxx}}=0,0<\alpha \leq 1
$$

şeklinde verilir. Burada $p$ ve $q$ keyfi sabitler, $\mathrm{D}_{\mathrm{t}}^{\alpha} u, \mathrm{t}$ zaman değişkenine göre $\alpha$ yıncı mertebeden conformable türevdir. Bu denklem üç boyutlu lineer olmayan dispersiyon problemlerinde önemli bir rol oynamaktadır. $\mathrm{Bu}$ denklem, soliton genișliğinin plazma homojenliğinin ölçek uzunluğuyla kıyaslandığında küçük olduğu varsayımıyla, pertürbasyon açılımlarından elde edilir. $\mathrm{Bu}$ varsayımda soliton, özeliğini, genliğini, genişliğini ve hızını korur. mKdV denklemi özel soliton yapısıyla bilinir. Esasen, genellikle karma KdV-mKdV denklemi olarak bilinen mKdV denklemi ya da Galilean dönüștürülmüş versiyonu birçok fiziksel olayda karşımıza çıkar. Bunlardan bazıları harmonik lattisler, Alfvóen dalgalar, iyonakustik solitonlar, trafik sıkışıklı̆̆ı, Schottky bariyer iletim hatları olarak sıralanabilir. mKdV denklemi, boyutlandırılmıș filmler elektrodinamik, elastik medya ve trafik akışındaki çok bileşenli plazmalar, elektrik devreleri, elektromanyetik dalgalarda karşımıza çıkar [14].

Denklem (7) ile verilen denklemi adi diferensiyel denkleme dönüştürmek için aşağıdaki dalga dönüşümü kullanılır.

$$
\mathrm{u}(\mathrm{x}, \mathrm{t})=\mathrm{u}(\xi), \xi=\mathrm{x}-l \frac{t^{\alpha_{1}}}{\alpha_{1}}
$$

Burada, $l$ sıfırdan farklı keyfi sabittir ve dalganın hızını göstermektedir. Böylelikle

$$
-l u^{\prime}+p u^{2} u^{\prime}+q u^{\prime \prime \prime}=0
$$

adi diferensiyel denklemi elde edilir. Bulunan adi diferensiyel denklemin $\xi$ ye göre bir kez integrali alınırsa;

$$
-l u+\frac{1}{3} p u^{3}+q u^{\prime \prime}=0
$$

adi diferensiyel denklemi bulunur. Buradan genelleştirilmiş Kudrayshov metoduna göre, (10) numaralı indirgenmiş denklemde en yüksek mertebeden türevli terim ile en yüksek dereceden lineer olmayan terimin dengelenmesiyle,

$$
N=M+1
$$

bağıntısı elde edilir. Burada $\mathrm{M}=1$ ve $\mathrm{N}=2$ seçilirek, (10) denkleminde çözüm,

$$
u(\xi)=\frac{a_{0}+a_{1} Q+a_{2} Q^{2}}{b_{0}+b_{1} Q}
$$

biçiminde aranır. Burada $Q(\xi)$ Denklem (5) denkleminin çözümüdür. (12) denkleminde $a_{0}, a_{1}, a_{2}, b_{0}, b_{1}$ daha sonra hesaplanacak parametrelerdir. Denklem (12), Denklem (5) yardımıyla Denklem (10) da yerine yazılırak, $Q^{\mathrm{k}} \mathrm{nın}$ kuvvetlerine göre paranteze alınıp ve $Q^{k} n ı n$ tüm katsayılarının sıfıra eşitlenmesiyle aşağıdaki denklem sistemi elde edilir.

$$
\begin{aligned}
& \mathrm{Q}^{6}: \quad 2 \mathrm{qa}_{2} \mathrm{~b}_{1}^{2}+(1 / 3) \mathrm{pa}_{2}^{3}=0, \\
& Q^{5}: \quad \mathrm{pa}_{1} \mathrm{a}_{2}^{2}-3 q \mathrm{q}_{2} \mathrm{~b}_{1}^{2}+6 q \mathrm{q}_{2} \mathrm{~b}_{0} \mathrm{~b}_{1}=0, \\
& Q^{4}: \quad-9 q a_{2} b_{0} b_{1}+p a_{1}^{2} a_{2}+q a_{2} b_{1}^{2}+6 q a_{2} b_{0}^{2}- \\
& l a_{2} b_{1}^{2}+p a_{0} a_{2}^{2}=0, \\
& Q^{3}: \mathrm{qa}_{1} \mathrm{~b}_{0} \mathrm{~b}_{1}+2 q \mathrm{q}_{1} \mathrm{~b}_{0}{ }^{2}+(1 / 3) p \mathrm{a}_{1}{ }^{3}-\mathrm{la}_{1} \mathrm{~b}_{1}{ }^{2}+ \\
& 2 \mathrm{pa}_{0} \mathrm{a}_{1} \mathrm{a}_{2}+3 q \mathrm{qa}_{2} \mathrm{~b}_{0} \mathrm{~b}_{1}-2 \mathrm{la}_{2} \mathrm{~b}_{0} \mathrm{~b}_{1}-10 q \mathrm{a}_{2} \mathrm{~b}_{0}{ }^{2} \\
& -2 q a_{0} b_{0} b_{1}-q a_{0} b_{1}^{2}=0, \\
& Q^{2}:-2 l a_{1} b_{0} b_{1}-q a_{1} b_{0} b_{1}-l a_{0} b_{1}{ }^{2}+p a_{0}{ }^{2} a_{2} \\
& +\mathrm{pa}_{0} \mathrm{a}_{1}^{2}+4 \mathrm{qa}_{2} \mathrm{~b}_{0}^{2}-3 \mathrm{qa}_{1} \mathrm{~b}_{0}+3 \mathrm{qa}_{0} \mathrm{~b}_{0} \mathrm{~b}_{1}-\mathrm{la}_{2} \mathrm{~b}_{0}^{2} \\
& +\mathrm{qa}_{0} \mathrm{~b}_{1}{ }^{2}=0 \text {, } \\
& Q^{1}:-l a_{1} b_{0}^{2}+q a_{1} b_{0}^{2}-2 l a_{0} b_{0} b_{1} \\
& -q a_{0} b_{0} b_{1}+p a_{0}^{2} a_{1}=0, \\
& \mathrm{Q}^{0}: \quad-\mathrm{la}_{0} \mathrm{~b}_{0}{ }^{2}+(1 / 3) \mathrm{pa}_{0}{ }^{3}=0 \text {. }
\end{aligned}
$$

Yukarıdaki cebirsel denklem sistemi Maple yardımıyla çözülürse, farklı 8 adet çözüm bulunur. Şekil 1-4 ile denklemin her bir durumda bulunan çözümlerinin grafiklerini verelim:

\section{Durum 1:}

$$
\begin{gathered}
a_{0}=0, a_{1}= \pm \sqrt{-\frac{3 q}{2 p}} b_{1}, a_{2}= \pm \frac{3 q b_{1}}{p \sqrt{-\frac{3 q}{2 p}}} \\
b_{0}=0, b_{1}=b_{1}, l=-\frac{q}{2} .
\end{gathered}
$$

Bulunan değerler Denklem (12) de yerine yazılırsa;

$$
\begin{aligned}
& \mathrm{u}_{1,2}(\mathrm{x}, \mathrm{t}) \\
& =\frac{ \pm \sqrt{-\frac{3 q}{2 p}} b_{1}\left(\frac{1}{1+A e^{\left(\mathrm{x}+\frac{q}{2} \cdot \frac{\alpha_{1}}{\alpha_{1}}\right)}}\right) \pm \frac{3 q b_{1}}{p \sqrt{-\frac{3 q}{2 p}}}\left(\frac{1}{\left.1+A e^{\left(\mathrm{x}+\frac{q}{2} \cdot t^{\alpha_{1}}\right.}\right)}\right)^{2}}{b_{1}\left(\frac{1}{\left.1+A e^{\left(\mathrm{x}+\frac{q}{2} \cdot \bar{\alpha}_{1}\right.}\right)}\right)}
\end{aligned}
$$

şeklinde 2 farklı çözüm bulunur. Burada A integral sabitidir. 


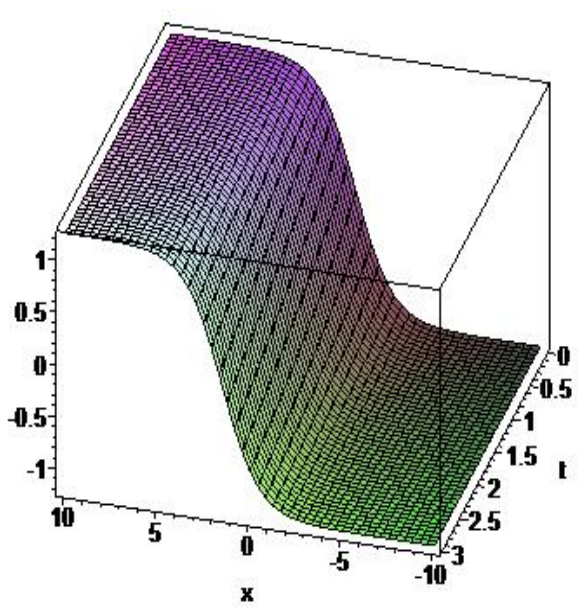

Şekil 1. $A=1, q=-1 \alpha_{1}=0.8, b_{1}=1, \mathrm{p}=1$

değerleri için $u(x, t)$ çözümüne karşılık gelen grafik

\section{Durum 2:}

$$
\begin{array}{r}
a_{0}=0, a_{1}= \pm 2 \sqrt{-\frac{6 q}{p}} b_{0}, a_{2}=\mp 2 \sqrt{-\frac{6 q}{p}} b_{0} \\
b_{0}=b_{0}, b_{1}=-2 b_{0}, l=q .
\end{array}
$$

Yukarıdaki değerler Denklem (12) de yerine yazılırsa;

$$
=\frac{ \pm 2 \sqrt{-\frac{6 q}{p}} b_{0}\left(\frac{1}{1+A e^{\left(\mathrm{x}-q \frac{t^{\alpha_{1}}}{\alpha_{1}}\right)}}\right) \mp 2 \sqrt{-\frac{6 q}{p}} b_{0}\left(\frac{1}{1+A e^{\left(\mathrm{x}-q \frac{t^{\alpha_{1}}}{\alpha_{1}}\right)}}\right)^{2}}{\mathrm{~b}_{0}-2 b_{0}\left(\frac{1}{1+A e^{\left(\mathrm{x}-q \frac{t^{\alpha_{1}}}{\alpha_{1}}\right)}}\right)}
$$

çözümleri bulunur. Burada A integral sabitidir.

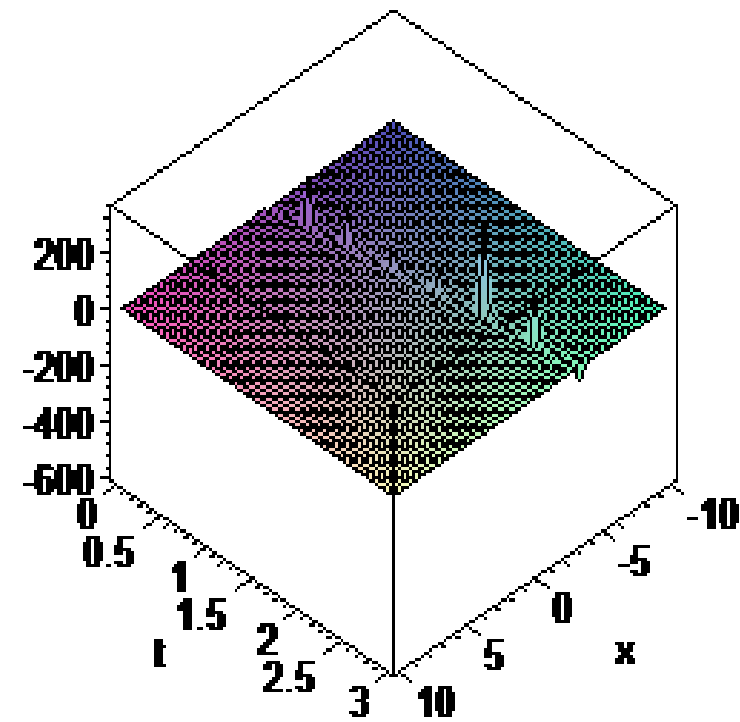

Şekil 2. $A=2, q=-1 \alpha_{1}=0.8, b_{0}=1, \mathrm{p}=1$

değerleri için $u(x, t)$ çözümüne karşıllk gelen grafik

\section{Durum 3:}

$$
\begin{gathered}
a_{0}=\mp \frac{3 q b_{1}}{\sqrt{-\frac{6 q}{p}} p}, a_{1}= \pm \frac{6 q b_{1}}{\sqrt{-\frac{6 q}{p}} p}, \\
a_{2}= \pm \sqrt{-\frac{6 q}{p}} b_{1}, b_{0}=-\frac{b_{1}}{2}, \\
b_{1}=b_{1}, l=-2 q .
\end{gathered}
$$

bulunur. Verilen denklemin çözümleri ise;

$$
\mathrm{u}_{5,6}(\mathrm{x}, \mathrm{t})=\frac{\mp \frac{3 q b_{1}}{\sqrt{-\frac{6 q}{p}} p} \pm \frac{6 q b_{1}}{\sqrt{-\frac{6 q}{p}} p}\left(\frac{1}{1+A e^{\left(\mathrm{x}+2 q \frac{t^{\alpha_{1}}}{\alpha_{1}}\right)}}\right)}{-\frac{b_{1}}{2}+\mathrm{b}_{1}\left(\frac{1}{1+A e^{\left(\mathrm{x}+2 q \frac{t^{\alpha_{1}}}{\alpha_{1}}\right)}}\right)}
$$

$$
\pm \frac{\sqrt{-\frac{6 q}{p}} b_{1}\left(\frac{1}{1+A e^{\left(\mathrm{x}+2 q \frac{t^{\alpha_{1}}}{\alpha_{1}}\right)}}\right) 2}{-\frac{b_{1}}{2}+\mathrm{b}_{1}\left(\frac{1}{1+A e^{\left(\mathrm{x}+2 q \frac{t^{\alpha_{1}}}{\alpha_{1}}\right)}}\right)}
$$

olarak elde edilir. Burada A integral sabitidir.

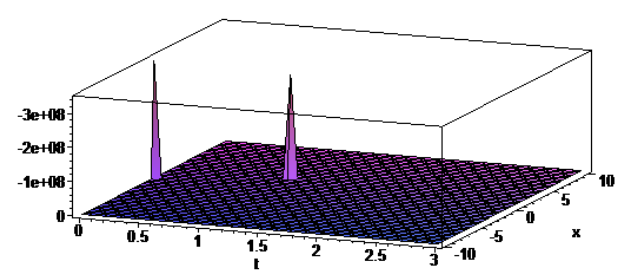

Şekil 3. $A=1, q=-1 \alpha_{1}=0.8, b_{1}=1, \mathrm{p}=1$

değerleri için $u(x, t)$ çözümüne karşıllk gelen grafik

\section{Durum 4:}

$$
\begin{gathered}
a_{0}= \pm \frac{3 q b_{0}}{\sqrt{-\frac{6 q}{p}} p}, a_{1}=\mp \frac{3 q\left(-b_{1}+2 b_{0}\right)}{\sqrt{-\frac{6 q}{p}} p}, a_{2}= \pm \sqrt{-\frac{6 q}{p}} b_{1} \\
b_{0}=b_{0}, b_{1}=b_{1}, l=-\frac{q}{2} .
\end{gathered}
$$

değerleri indirgenmiş adi diferensiyel denklemde yerine yazılırsa;

$$
\mathrm{u}_{7,8}(\mathrm{x}, \mathrm{t})=\frac{ \pm \frac{3 q b_{0}}{\sqrt{-\frac{6 q}{p}} p} \mp \frac{3 q\left(-b_{1}+2 b_{0}\right)}{\sqrt{-\frac{6 q}{p}} p}\left(\frac{1}{1+A e^{\left(\mathrm{x}+\frac{\mathrm{q} t^{\alpha_{1}}}{2 \alpha_{1}}\right)}}\right)^{2}}{\mathrm{~b}_{0}+\mathrm{b}_{1}\left(\frac{1}{1+A e^{\left(\mathrm{x}+\frac{\mathrm{q} t^{\alpha_{1}}}{2 \alpha_{1}}\right)}}\right)}
$$




$$
\pm \frac{\sqrt{-\frac{6 q}{p}} b_{1}\left(\frac{1}{1+A e^{\left(\mathrm{x}+\frac{\mathrm{q} t^{\alpha_{1}}}{2 \alpha_{1}}\right)}}\right)^{2}}{\mathrm{~b}_{0}+\mathrm{b}_{1}\left(\frac{1}{1+A e^{\left(\mathrm{x}+\frac{\mathrm{q} \mathrm{t}^{\alpha_{1}}}{2 \alpha_{1}}\right)}}\right)}
$$

çözümleri elde edilir. Burada A integral sabitidir.

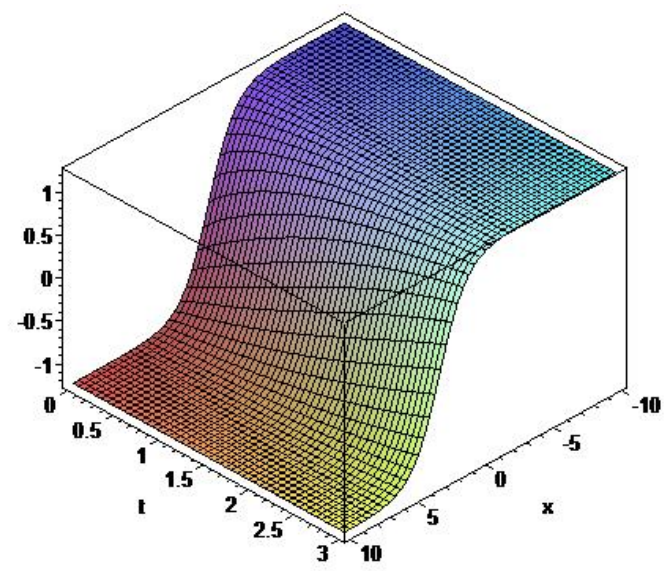

Şekil 4. $A=1, q=-2 \alpha_{1}=0.8, b_{0}=1, \mathrm{p}=2$

değerleri için $u(x, t)$ çözümüne karşılık gelen grafik

\subsection{Conformable Boussinesq denklemi}

Conformable Boussinesq denklemi

$$
\frac{\partial^{2 \alpha} \mathrm{u}(\mathrm{x}, \mathrm{t})}{\partial \mathrm{t}^{2 \alpha}}-\frac{\partial^{2} \mathrm{u}(\mathrm{x}, \mathrm{t})}{\partial \mathrm{x}^{2}}-\frac{\partial^{2} \mathrm{u}^{2}(\mathrm{x}, \mathrm{t})}{\partial \mathrm{x}^{2}}+\frac{\partial^{4} \mathrm{u}(\mathrm{x}, \mathrm{t})}{\partial \mathrm{x}^{4}}
$$

şeklinde verilir ve $0<\alpha \leq 1$ dir. Bu denklem yatay ölçeği suyun derinliğinden çok daha büyük olan yüzey dalgalarını tanımlar $[15,16]$.

Denklem (14) ;

$$
\mathrm{u}(\mathrm{x}, \mathrm{t})=\mathrm{u}(\xi), \xi=\mathrm{x}-l \frac{t^{\alpha_{1}}}{\alpha_{1}}
$$

dalga dönüşümü kullanılarak

$$
\left(1^{2}-1\right) u^{\prime \prime}-\left(u^{2}\right)^{\prime \prime}+u^{(4)}=0
$$

adi diferensiyel denklemi elde edilir. Bulunan adi diferensiyel denklemin iki defa $\xi$ ye göre integrali alınırsa ve integral sabitleri sıfıra eşitlenirse

$$
\mathrm{u}^{\prime \prime}+\left(\mathrm{l}^{2}-1\right) \mathrm{u}-\mathrm{u}^{2}=0(16)
$$

adi diferensiyel denklemi bulunur. (16) denkleminde genelleştirilmiş Kudryashov yöntemine göre $\mathrm{N}=\mathrm{M}+2$ elde edilir. Burada $\mathrm{M}=1$ ve $\mathrm{N}=3$ seçilmesiyle (16) denkleminin analitik çözümleri, (4) numaralı denkleme göre,

$$
u(\xi)=\frac{a_{0}+a_{1} Q+a_{2} Q^{2}+a_{3} Q^{3}}{b_{0}+b_{1} Q}
$$

olarak aranır. Burada $Q(\xi),(5)$ denkleminin çözümü ve $a_{0}, a_{1}, a_{2}, a_{3}, b_{0}, b_{1}$ daha sonra hesaplanacak parametrelerdir. (17) numaralı denklemin (16) numaralı denklemde yerine yazılıp, (5) numaralı denklemin kullanılmasıyla elde edilen denklemin $Q^{k}$ nın kuvvetlerine göre düzenlenmesi ve $Q^{k}$ terimlerinin başındaki bütün katsayıların sıfıra eşitlenmesiyle aşağıdaki denklem sistemi elde edilir.

$$
\begin{aligned}
& Q^{7}: \quad 6 a_{3} b_{1}^{2}-a_{3}^{2} b_{1}=0, \\
& Q^{6}: 2 a_{2} b_{1}^{2}-2 a_{2} a_{3} b_{1}-10 a_{3} b_{1}^{2} \\
& +16 a_{3} b_{0} b_{1}-a_{3}^{2} b_{0}=0 \text {, } \\
& Q^{5}: 3 a_{3} b_{1}^{2}+6 a_{2} b_{0} b_{1}-2 a_{2} a_{3} b_{0}+ \\
& 12 a_{3} b_{0}^{2}-2 a_{1} a_{3} b_{1}-a_{2}^{2} b_{1} \\
& -3 a_{2} b_{1}^{2}+l^{2} a_{3} b_{1}^{2}-27 a_{3} b_{0} b_{1}=0, \\
& Q^{4}:-9 a_{2} b_{0} b_{1}+l^{2} a_{2} b_{1}^{2}+2 l^{2} a_{3} b_{0} b_{1} \\
& +6 a_{2} b_{0}^{2}-21 a_{3} b_{0}^{2}-2 a_{1} a_{3} b_{0} \\
& -2 a_{1} a_{3} b_{0}-2 a_{0} a_{3} b_{1}+9 a_{3} b_{0} b_{1} \\
& -\mathrm{a}_{2}^{2} \mathrm{~b}_{0}-2 \mathrm{a}_{1} \mathrm{a}_{2} \mathrm{~b}_{1}=0 \text {, } \\
& Q^{3}: a_{1} b_{0} b_{1}-2 b_{1} a_{0} b_{0}+l^{2} a_{3} b_{0}^{2}+8 a_{3} b_{0}^{2} \\
& -2 a_{1} a_{2} b_{0}-2 a_{0} a_{2} b_{1}-2 a_{0} a_{3} b_{0}+a_{2} b_{0} b_{1} \\
& +l^{2} a_{1} b_{1}^{2}-a_{1} b_{1}^{2}-a_{1}^{2} b_{1}+2 a_{1} b_{0}^{2} \\
& +2 \mathrm{l}^{2} \mathrm{a}_{2} \mathrm{~b}_{0} \mathrm{~b}_{1}-10 \mathrm{a}_{2} \mathrm{~b}_{0}^{2}-\mathrm{b}_{1}^{2} \mathrm{a}_{0}=0, \\
& Q^{2}:-2 a_{0} a_{2} b_{0}+2 l^{2} a_{1} b_{0} b_{1}+l^{2} a_{2} b_{0}^{2}+3 b_{1} a_{0} b_{0} \\
& -2 a_{0} a_{1} b_{1}+l^{2} a_{0} b_{1}^{2}-a_{1}^{2} b_{0} \\
& -3 a_{1} b_{0}^{2}-3 a_{1} b_{0} b_{1}+3 a_{2} b_{0}^{2}=0 \text {, } \\
& Q^{1}: 2 l^{2} a_{0} b_{0} b_{1}-3 b_{1} a_{0} b_{0}-2 a_{0} a_{1} b_{0} \\
& -a_{0}^{2} b_{1}+l^{2} a_{1} b_{0}^{2}=0 \text {, }
\end{aligned}
$$$$
\mathrm{Q}^{0}: \quad-\mathrm{a}_{0} \mathrm{~b}_{0}^{2}-\mathrm{a}_{0}^{2} \mathrm{~b}_{0}+\mathrm{l}^{2} \mathrm{a}_{0} \mathrm{~b}_{0}^{2}=0
$$

Yukarıdaki cebirsel denklem sistemi Maple yardımıyla çözülürse; 


$$
\begin{gathered}
a_{0}=b_{0}, a_{1}=b_{1}-6 b_{0}, a_{2}=-6 b_{1}+6 b_{0}, \\
a_{3}=6 b_{1}, b_{0}=b_{0}, b_{1}=b_{1}, l=\sqrt{2}
\end{gathered}
$$

olarak bulunur. 0 halde, conformable Boussinesq denkleminin çözümü

$$
\begin{aligned}
& \mathrm{u}(\mathrm{x}, \mathrm{t}) \\
& =\frac{\mathrm{b}_{0}+\left(b_{1}-6 b_{0}\right) \mathrm{Q}+\left(-6 b_{1}+6 b_{0}\right) \mathrm{Q}^{2}+\left(6 b_{1}\right) \mathrm{Q}^{3}}{\mathrm{~b}_{0}+\mathrm{b}_{1} \mathrm{Q}}
\end{aligned}
$$

olarak bulunur. Burada

$$
Q=\left(\frac{1}{1+A e^{\left(\mathrm{x}-\sqrt{2} \frac{t^{\alpha_{1}}}{\alpha_{1}}\right)}}\right) \operatorname{dir} .
$$

Yukarıda bulmuş olduğumuz çözümde gerekli seçimleri yaptığımızda Şekil 5 ile verilen grafiği elde ederiz.

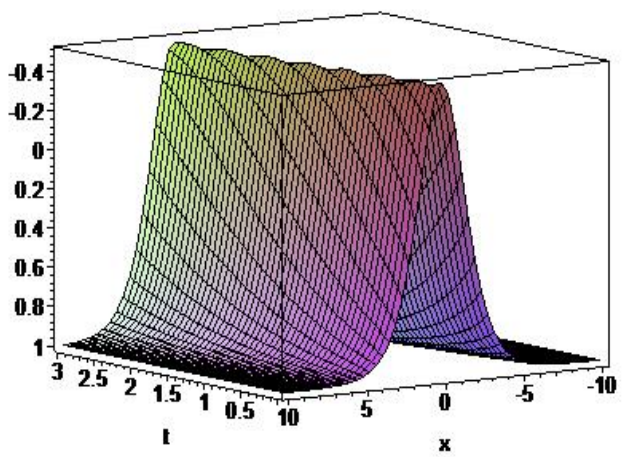

Şekil 5. $A=1, \alpha_{1}=0.8$ değerleri için $u(x, t)$ çözümüne karşıllk gelen grafik

\section{Tartışma ve Sonuç}

Bu çalışmada özet olarak genelleştirilmiş Kudryashov metodu lineer olmayan denklemlerin tam çözümlerini bulabilmek için tanıtılmıștır. Conformable üçüncü mertebeden modifiye $\mathrm{KdV}$ denklemi ve conformable Boussinesq denkleminin analitik çözümlerini bu verilen metot ile elde edilmiştir. Ayrıca elde edilen çözümlerin grafiklerine yer verilmiştir.

Conformable Üçüncü Mertebeden Modifiye KdV denklemin altı adet tam çözümü Sahoo ve Ray tarafından (G'/G)-açılım yöntemi ve geliștirilmiș (G'/G) yöntemi kullanılarak elde edilmiştir [14]. Akbulut ve Kaplan tarafından yardımcı denklem metodu ile bu denklemin çözümleri elde edilmiştir [15]. Sabi'u ve çalışma arkadaşları tarafından sinüscosinüs yöntemiyle çeşitli çözümler elde edilmiştir [16]. Conformable Boussinesq denklemi ise Hosseini ve Ansari'nin çalışmasında ele alınmış ve modifiye edilmiş Kudryshov yöntemi ile tam çözümleri bulunmuştur [16]. Ayrıca Hosseini ve çalışma arkadaşları tarafından $\exp (-\phi(\varepsilon))$-expansion yöntemiyle ise farklı çözümler elde edilmiştir [19]. Bu çalışmada elde edilen çözümleri diğer çalışmalarda elde edilenlerle kıyaslarsak, parametrelerin uygun seçimi ile diğer çalışmalarda benzer çözümler elde edilebilecek iken, bu çalışmada elde edilen çözümlerin yine farklı olduğu görülür. Sözü geçen tüm yöntemlerde yardımcı denklem yardımıyla çözümlere ulaşılmaktadır, Ayrıca genelleştirilmiş Kudryshov yöntemi diğer yöntemlere kiyasla uygulanması daha pratik bir yöntemdir.

Bir başka deyişle, genelleştirilmiş Kudryashov metodu lineer olmayan diferensiyel denklemlerin tam çözümlerini bulmak için etkili ve güçlü bir yöntemdir. Matematiksel fizik ve lineer olmayan bilimlerde kullanılan lineer olmayan denklemlerin analitik çözümlerinin bulunmasında kolaylıkla uygulanabilir bir yöntemdir.

$\mathrm{Bu}$ çalışmada elde edilen çözümlerin doğruluğu, Maple paket programı yardımıyla elde edilen çözümlerin yerine konulmasıyla teyit edilmiştir.

\section{Etik Beyanı}

Bu çalışmada, "Yükseköğretim Kurumları Bilimsel Araştırma ve Yayın Etiği Yönergesi" kapsamında uyulması gerekli tüm kurallara uyulduğunu, bahsi geçen yönergenin "Bilimsel Araştırma ve Yayın Etiğine Aykırı Eylemler" başlığı altında belirtilen eylemlerden hiçbirinin gerçekleştirilmediğini taahhüt ederiz.

\section{Kaynakça}

[1] Ablowitz, M. J., Segur, H. 1981. Solitons and Inverse Scattering Transformation, SIAM, Philadelphia, 438 s.

[2] Wang, M. L. 1995. Solitary Wave Solutions for Variant Boussinesq Equations. Physics Letters A, 199, 169- 172.

[3] Wazwaz, A. M. 2007. Multiple-Soliton Solutions for the Boussinesq Equation. Applied Mathematics and Computation, 192(2), 479-486.

[4] Gümüş, H., Yılmaz, H. 2019. Nonlineer Schrödinger Denkleminin Tam Çözümleri. Türkiye Teknoloji ve Uygulamalı Bilimler Dergisi, 2(1), 11-19.

[5] Gurefe, Y., Misirlı, E. 2011. Exp-function Method for Solving Nonlinear Evolution Equations with Higher Order Nonlinearity. Computers\& Mathematics with Applications, 61 (8), 20252030.

[6] Gurefe, Y., Mısırlı, Pandir, Y., Sönmezoğlu, A., Ekici, M. 2015. New Exact Solutions of the Davey-Stewartson Equation with Power-Law Nonlinearity. The Bulletin of the Malaysian Mathemetical Society Series, 38(3), 1223-1234.

[7] Bulut, H., Pandir, Y., Tuluce Demiray, S. 2014. Exact Solutions of Nonlinear Schrodinger's Equation with Dual PowerLaw Nonlinearity by 
Extended Trial Equation Method. Waves Random Complex Media, 24(4), 439-451.

[8] Tasbozan, O., Kurt, A. 2020. The New Travelling Wave Solutions of Time Fractional FitzhughNaguma Equation with Sine-Gordon Expansion Method. Adıyaman Üniversitesi Fen Bilimleri Dergisi, 10(1), 256-263.

[9] Başkonuş, H. M., Bulut, H., Tukur, A. S. 2017. Investigation of Various Travelling Wave Solutions to the Extended $(2+1)$-dimensional Quantum ZK Equation. The European Physical Journal Plus, 132, 482-490.

[10] Demiray, S. T. 2019. New Exact Solutions of (3+1)-Dimensional Modified Quantum Zakharov-Kuznetsov Equation. Turkish Journal of Mathematics and Computer Science, 11, 5659.

[11] Khalil, R., Horani, M. Al., Yousef, A., Sababbeh, M. 2014. A new definition of fractional derivative, Journal of Computational and Applied Mathematics, 264, 65-70.

[12] Kudryashov, N. A. 2013. Polynomials in Logistic Function and Solitary Waves of Nonlinear Differential Equations. Applied Mathematics and Computation, 219(17), 9245-9253.

[13] Kaplan, M., Bekir, A., Akbulut, A. 2016. A generalized Kudryashov to Some Nonlinear Evolution Equations in Mathematical Physics. Nonlinear Dynamics, 85(4), 2843-2850.
[14] Sahoo, S., Ray, S. 2016. Solitary Wave Solutions for Time Fractional Third Order Modified KdV Equation Using Two Reliable Techniques ( $\left.G^{\prime} / G\right)$ Expansion Method and improved (G'/G)Expansion Method. Physica A: Statistical Mechanics and Its Applications, 448, 265-282.

[15] Wazwaz, A. M. 2001. Construction of Soliton Solutions and Periodic Solutions of the Boussinesq Equation by the Modified Decomposition Method. Chaos, Soliton and Fractals, 12, 1549-1556.

[16] Hosseini, K., Ansari, R. 2017. New Exact Solutions of Nonlinear Conformable TimeFractional Boussinesq Equations Using the Modified Kudryashov Method. Waves in Random and Complex Media, 27(4), 628-636.

[17] Akbulut, A., Kaplan, M., 2018. Auxiliary equation method for time-fractional differential equations with conformable derivative, Computers \& Mathematics with Applications, 75(3), 876-882.

[18] Sabi'u, J., Jibril, A., Gadu, A. M. 2019. New exact solution for the $(3+1)$ conformable space-time fractional modified Korteweg-de-Vries equations via Sine-Cosine Method, Journal of Taibah University Science, 13(1), 91-95.

[19] Hosseini K., Bekir A., Ansari R. 2017. Exact solutions of nonlinear conformable timefractional Boussinesq equations using the $\exp (-$ $\phi(\varepsilon))$-expansion Method, Opt Quant Electron 49, 131. 